\title{
DESIGN OF WIDEBAND MIMO RADAR WAVEFORMS WITH LOW PEAK-TO-AVERAGE RATIO
}

\author{
Yonghao Tang ${ }^{1,2}$, Yimin D. Zhang ${ }^{2}$, Moeness G. Amin ${ }^{2}$, Weixing Sheng ${ }^{1}$ \\ ${ }^{1}$ School of Electronic and Optical Engineering, Nanjing University of Science and Technology, China \\ ${ }^{2}$ Center for Advanced Communications, Villanova University, USA
}

\begin{abstract}
Multiple-input multiple-output (MIMO) radar systems allow array antennas to transmit different waveforms and, as such, enable flexible transmit beampattern synthesis. Most existing transmit beampattern synthesis methods focus on narrowband MIMO radar systems. In this paper, we propose a novel method to design transmit waveforms for wideband MIMO radar systems. The proposed technique is based on the optimization of the cross-spectral density matrix and achieves a low peak-to-average power ratio (PAR) as desired in practical radar operations. Simulations results are provided to verify the low PAR waveform design capability corresponding to arbitrary beampatterns.
\end{abstract}

Index Terms-Wideband MIMO radar, beampattern synthesis, waveform design, peak-to-average power ratio

\section{INTRODUCTION}

Optimized multiple-input multiple-output (MIMO) radar waveform design is key to achieving desirable transmit beampatterns and has attracted considerable interest in the radar community [1-4]. The majority of existing literature on MIMO radar waveform design focuses on narrowband signals [5-14]. These methods can be classified into two major categories. Methods in the first category, such as beampattern matching design and minimum sidelobe design, directly optimize the cross-correlation matrix of the transmit waveforms to synthesize desired transmit beampatterns $[5,8$, 9]. As transmit beampattern is a linear function of the correlation matrix, the formulated optimization problems can be easily solved to obtain the desired beampatterns. Methods in the other category design actual transmit waveforms based on a given cross-correlation matrix. Partially correlated signal design methods are also developed for narrowband MIMO radars [13, 14], while orthogonal signals are transmitted in sub-array MIMO radars $[10,11]$.

Studies on transmit beampattern synthesis for wideband MIMO radar systems, on the other hand, are still very limited [15-17]. Inspired by the methods developed for narrowband MIMO radar systems, wideband transmit beampattern synthesis through optimizing the cross-spectral density matrix (CSDM) was first proposed in [15]. As the CSDM optimization is often constructed as a convex optimization problem that can be conveniently solved, such approach enables flexible design of beampatterns. Some beampattern design methods based on CSDM optimization, such as those through beampattern matching and sidelobe minimization, were introduced in wideband MIMO radar beamforming $[15,17]$. To the authors' best knowledge, however, such results have not yet led to the wideband MIMO waveform design based on the optimized CSDM. Several other methods are developed for the design of wideband MIMO waveforms outside the CSDM context. For example, waveforms designed by the Spectral density Focusing Beampattern synthesis Technique (SFBT) [16] do not take the peak-to-average ratio (PAR) into account and thus may not be the desirable choice in practice applications. The Wideband Beampattern Formation via Iterative Technique (WBFIT) [18] links the beampattern to signals through the Fourier transform. The Fast OneDimensional Frequency Invariant Wideband Transmit Beampattern (F1D-FIWTB) method [19] designs waveforms through the Fourier transform by exploiting the frequency invariant beamforming (FIB) method developed in [20]. However, as these beampattern design methods are not based on CSDM optimization, the resulting beampatterns cannot account for other desired constraints, such as achieving low sidelobes and simultaneously synthesizing multiple beams at different frequencies.

In this paper, we optimize the CSDM and synthesize the corresponding transmit waveforms in the wideband MIMO radar context. The proposed approach enables flexible design of the transmit beampatterns and achieves a low PAR. We first briefly introduce beampattern design methods to optimize transmit beampatterns based on CSDM. Then, the proposed novel waveforms design algorithm is described for the synthesis of wideband beampatterns. Simulations results are provided to verify the low PAR waveform design capability corresponding to arbitrary beampatterns.

Notations: we use lower-case (upper-case) bold characters to denote vectors (matrices). $(\cdot)^{*}$ stands for complex conjugation, and $\operatorname{Re}(\cdot)$ denotes the real part of a complex value. $(\cdot)^{T}$ and $(\cdot)^{H}$, respectively, denote transpose and conjugate transpose of a matrix or vector. trace $(\cdot)$ implies matrix trace. $\boldsymbol{A} \succeq 0$ means that $\boldsymbol{A}$ is a positive semi-definite matrix. In addition, $\mathrm{E}(\cdot)$ denotes statistical expectation. 


\section{SIGNAL MODEL AND CSDM OPTIMIZATION}

\subsection{Signal Model}

Consider a uniform linear array (ULA) consisting of $M$ omnidirectional antennas with an inter-element spacing of $d$. Denote the signal transmitted by the $m$ th antenna as $s_{m}(t)=x_{m}(t) e^{j 2 \pi f_{c} t}, m=1, \ldots, M$, where $f_{c}$ is the carrier frequency and $x_{m}(t)$ is the baseband waveforms occupying a wide spectral band of $[-B / 2, B / 2]$. In the far field, the total received signal can be expressed as

$$
\hat{s}(t)=\sum_{m=1}^{M} s_{m}\left(t-\tau_{m}\right),
$$

where $\tau_{m}$ denotes the time delay between the $m$ th antenna and the reference one. Therefore, the total signal power due to all signals is given by [15]

$$
E\left\{\|\hat{s}(t)\|^{2}\right\}=\sum_{i=1}^{M} \sum_{j=1}^{M} R_{i j}\left(\tau_{i}-\tau_{j}\right),
$$

where

$$
\begin{gathered}
R_{i j}\left(\tau_{i}-\tau_{j}\right)=0.5 \operatorname{Re}\left\{\bar{R}_{i j}\left(\tau_{i}-\tau_{j}\right) e^{-j 2 \pi f_{c}\left(\tau_{i}-\tau_{j}\right)}\right\}, \\
\bar{R}_{i j}\left(\tau_{i}-\tau_{j}\right)=0.5 \mathrm{E}\left\{x_{i}(t) x_{j}{ }^{*}\left(t+\tau_{i}-\tau_{j}\right)\right\} .
\end{gathered}
$$

Stacking the correlation function of $x_{m}(t)$ for all sensor pairs yields the following correlation matrix

$$
\overline{\boldsymbol{R}}(\tau)=\left[\begin{array}{cccc}
\bar{R}_{11}(\tau) & \bar{R}_{21}(\tau) & \cdots & \bar{R}_{M 1}(\tau) \\
\bar{R}_{12}(\tau) & \bar{R}_{22}(\tau) & \cdots & \bar{R}_{M 2}(\tau) \\
\vdots & \vdots & \ddots & \vdots \\
\bar{R}_{1 M}(\tau) & \bar{R}_{2 M}(\tau) & \cdots & \bar{R}_{M M}(\tau)
\end{array}\right] .
$$

\subsection{CSDM based Beampattern Optimization}

For narrowband signals, because $\bar{R}_{i j}(\tau) \approx \bar{R}_{i j}(0)$, the spatial power distribution becomes [15],

$$
P\left(\theta, f_{c}\right)=\boldsymbol{a}^{H}\left(\theta, f_{c}\right) \boldsymbol{R} \boldsymbol{a}\left(\theta, f_{c}\right),
$$

where $\boldsymbol{a}\left(\theta, f_{c}\right)$ is the narrowband array steering vector, $\theta$ is the spatial angle, and $\boldsymbol{R}=\overline{\boldsymbol{R}}(0)$. However, in the wideband case, the spatial power distribution becomes frequencydependent. The CSDM is defined as [15],

$$
\boldsymbol{S}(f)=\int \overline{\boldsymbol{R}}(\tau) e^{-j 2 \pi f \tau} d \tau
$$

and the corresponding power pattern can be expressed as

$$
P(\theta)=\int_{-\frac{B}{2}}^{\frac{B}{2}} \boldsymbol{a}^{H}\left(\theta, f_{c}+f\right) \boldsymbol{S}(f) \boldsymbol{a}\left(\theta, f_{c}+f\right) d f,
$$

where $\boldsymbol{a}\left(\theta, f_{c}+f\right)$ is the array steering vector at frequency $f_{c}+f$. Divide the spectral band $\left[f_{c}-B / 2, f_{c}+B / 2\right]$ into $N$ samples, denoted as $f_{n}, n=-N / 2, \ldots, N / 2-1$, where, without loss of generality, we assume $N$ to be an even integer for notational convenience. The spatial angle region $[-\pi / 2, \pi / 2]$ is divided into a $K$-point uniform grid with the $k$ th entry denoted as $\theta_{k}, k=1, \ldots, K$. The power distribution at spatial angle $\theta_{k}$ and frequency $f_{n}$ can then be written as

$$
p\left(\theta_{k}, f_{n}\right)=\boldsymbol{a}^{H}\left(\theta_{k}, f_{n}\right) \boldsymbol{S}\left(f_{n}\right) \boldsymbol{a}\left(\theta_{k}, f_{n}\right) .
$$

From eq. (9), it is clear that, at each frequency $f_{n}$, we can appropriately choose the CSDM $S\left(f_{n}\right)$ to design the transmit beampattern in a similar manner to the narrowband case. Similar to the narrowband MIMO radar beampattern design described in [5], the wideband MIMO radar beampattern can also be designed by optimizing the CSDM $\boldsymbol{S}\left(f_{n}\right)$ to achieve low sidelobe beampatterns [17]. The beampattern sidelobe level can be minimized by solving the following semi-definite programming problem (SDP)

$$
\min _{\left\{S\left(f_{n}\right)\right\}, q}-q
$$

s.t. $\quad \boldsymbol{S}\left(f_{n}\right) \succeq 0$,

$$
\begin{array}{r}
\operatorname{trace}\left[\boldsymbol{S}\left(f_{n}\right)\right]=1, \\
\boldsymbol{a}^{H}\left(\theta_{0}, f_{n}\right) \boldsymbol{S}\left(f_{n}\right) \boldsymbol{a}\left(\theta_{0}, f_{n}\right)-\boldsymbol{a}^{H}\left(\theta_{k}, f_{n}\right) \boldsymbol{S}\left(f_{n}\right) \boldsymbol{a}\left(\theta_{k}, f_{n}\right) \geq q, \\
\theta_{k} \in \boldsymbol{\Theta}, n=-N / 2, \ldots, N / 2-1, \\
\boldsymbol{a}^{H}\left(\theta_{l}, f_{n}\right) \boldsymbol{S}\left(f_{n}\right) \boldsymbol{a}\left(\theta_{l}, f_{n}\right)=0.5 \boldsymbol{a}^{H}\left(\theta_{0}, f_{n}\right) \boldsymbol{S}\left(f_{n}\right) \boldsymbol{a}\left(\theta_{0}, f_{n}\right), \\
\boldsymbol{a}^{H}\left(\theta_{r}, f_{n}\right) \boldsymbol{S}\left(f_{n}\right) \boldsymbol{a}\left(\theta_{r}, f_{n}\right)=0.5 \boldsymbol{a}^{H}\left(\theta_{0}, f_{n}\right) \boldsymbol{S}\left(f_{n}\right) \boldsymbol{a}\left(\theta_{0}, f_{n}\right),
\end{array}
$$

where $q$ is an auxiliary variable, $\boldsymbol{\Theta}$ is the sidelobe regions, $\theta_{0}$ represents the main beam direction, and $\theta_{l}$ and $\theta_{r}$ are respectively the lower and upper angles corresponding to the $3 \mathrm{~dB}$ beam-width at each frequency. The above constraints require a feasible solution to have a semidefinite CSDM at every discrete frequency. Additionally, the transmit energy at each frequency is set to unity with a total energy of $N$. Other CSDM optimization methods, such as beampattern matching design in [15], are also developed for wideband MIMO radar beamforming, in which we can also arbitrarily choose the desired beampattern to synthesize an approximate beampattern for wideband MIMO radar.

\section{LOW PAR WAVEFORMS DESIGN METHOD}

In narrowband MIMO radar, transmit waveforms can be designed according to a given correlation matrix $\mathbf{R}[5,8,9]$. For wideband MIMO radars, however, transmit waveform design based on the correlation function matrix $\overline{\boldsymbol{R}}(\tau)$ becomes more complicated as it involves different lags. In the following, we propose an alternative approach to design low PAR waveforms that approximately satisfy a given CSDM. The problem is first considered for the case where the CSDM is rank-one, and then a general case for a higher-rank CSDM is considered.

\subsection{Single-rank Case}

We first consider the simple case where the CSDM $\boldsymbol{S}\left(f_{n}\right)$ at each frequency has a single primary eigenvalue, whereas the other eigenvalues are negligible. In this case, $\boldsymbol{S}\left(f_{n}\right)$ can be expressed as

$$
\boldsymbol{S}\left(f_{n}\right)=\boldsymbol{y}_{n} \boldsymbol{y}_{n}^{H},
$$

where $\boldsymbol{y}_{n}$ represents the primary eigenvector. Note that $\boldsymbol{y}_{n}$ is 
subject to a group phase ambiguity because $\boldsymbol{y}_{n} \varphi_{n} \varphi_{n}{ }^{*} \boldsymbol{y}_{n}{ }^{H}=$ $\boldsymbol{y}_{n} \boldsymbol{y}_{n}{ }^{H}=\boldsymbol{S}\left(f_{n}\right)$ for any $\varphi_{n}$. Performing eigen-decomposition of $\boldsymbol{S}\left(f_{n}\right)$ for each frequency $f_{n}, n=-N / 2, \ldots, N / 2-1$, we express the transmit waveforms in the frequency domain as

$$
\boldsymbol{Y}=\left[\boldsymbol{y}_{-N / 2}, \boldsymbol{y}_{-N / 2+1}, \ldots, \boldsymbol{y}_{N / 2-1}\right]^{T}=\left[\tilde{\boldsymbol{y}}_{1}, \tilde{\boldsymbol{y}}_{2}, \ldots, \tilde{\boldsymbol{y}}_{M}\right]^{T},
$$

where $\tilde{\boldsymbol{y}}_{m}{ }^{T}$ denotes the $m$ th row of $\boldsymbol{Y}$. The $N$-symbol transmit sequence corresponding to each $\tilde{\boldsymbol{y}}_{m}$ can then be computed through inverse discrete Fourier transform (IDFT).

As the waveform is independently optimized in each frequency, the yielding waveforms that combine all the frequency components will have a high PAR. Maintaining a low PAR is important in practice to minimize energy loss and signal distortions. For this purpose, the low PAR waveform design problem is described as

$$
\begin{gathered}
\min _{\boldsymbol{X}, \boldsymbol{\varphi}}\|\boldsymbol{Z}-\boldsymbol{X}\|_{F} \\
\text { s.t. } \operatorname{PAR}\left(\boldsymbol{x}_{m}\right) \leq \rho, \text { for each } m, \\
\left\|\boldsymbol{x}_{m}\right\|_{2}^{2}=E_{0}, \text { for each } m,
\end{gathered}
$$

where

$$
\begin{aligned}
\boldsymbol{Z} & =\left[\tilde{\boldsymbol{z}}_{1}, \tilde{\boldsymbol{z}}_{2}, \ldots, \tilde{\boldsymbol{z}}_{M}\right]^{T}, \\
\tilde{\boldsymbol{z}}_{m} & =\operatorname{IDFT}\left[\operatorname{diag}(\boldsymbol{\varphi}) \tilde{\boldsymbol{y}}_{m}\right], \\
\boldsymbol{X} & =\left[\boldsymbol{x}_{1}, \boldsymbol{x}_{2}, \ldots, \boldsymbol{x}_{M}\right]_{M \times N}^{T},
\end{aligned}
$$

$\operatorname{IDFT}(\cdot)$ is the IDFT operator, $\varphi=\left[\varphi_{-N / 2}, \ldots, \varphi_{n}, \ldots, \varphi_{N / 2-1}\right]$ represents the aforementioned group phase ambiguties. In addition, $E_{0}$ is the energy transmitted from each transmitter, and $\rho \geq 1$ is the maximum permissible PAR of the $m$ th sequence, which is defined as

$$
\operatorname{PAR}\left(\boldsymbol{x}_{m}\right)=\frac{N \max _{n}\left|\boldsymbol{x}_{m}(n)\right|^{2}}{\sum_{n}\left|\boldsymbol{x}_{m}(n)\right|^{2}} .
$$

Note that $\rho=1$ implies that the resulting waveforms are constant modulus.

Because of the complex expression (17), the minimization in (13) with respect to $\boldsymbol{X}$ and $\boldsymbol{\varphi}$ does not have a closed-form solution. However, several local optimal solutions with respect to either $\boldsymbol{X}$ or $\boldsymbol{\varphi}$ dimension are available. In the proposed iterative algorithm, this optimization problem is solved by iteratively updating the individual optimization problems with respect to the transmit waveforms $\boldsymbol{X}$ and the phase ambiguities $\boldsymbol{\varphi}$. This kind of optimization techniques has been applied in other fields [21, $22]$, so that the convergence of the proposed method can be guaranteed. The computation complexity of the proposed algorithm is $O\left(M^{3}+L M N\left(N+\log _{2} N\right)\right)$, where $L$ is the number of iterations. The proposed iterative algorithm is summarized in Table I.

\subsection{General Case}

In this subsection, we extend the proposed method to the general case where the CSDM $\boldsymbol{S}\left(f_{n}\right)$ has a higher rank. In this case, the number of the primary eigenvalues of $\boldsymbol{S}\left(f_{n}\right)$

\section{Table I: Proposed Iterative Algorithm}

Step 1. Apply eigen-decomposition to the given $\boldsymbol{S}\left(f_{n}\right)$, initialize $\boldsymbol{\varphi}=\left[e^{j 0}, \ldots, e^{j 0}\right]$, and compute $\boldsymbol{Y}$ and $\boldsymbol{Z}$ using eqs. (12), (14), and (15);

Step 2. For each $m, m=1, \ldots, M$, solve the nearest-vector problems [23] to obtain $\boldsymbol{x}_{m}$ based on $\boldsymbol{\varphi}$ :

$$
\begin{gathered}
\min _{\boldsymbol{x}_{m}}\left\|\tilde{\boldsymbol{z}}_{m}-\boldsymbol{x}_{m}\right\|_{2} \\
\text { s.t. } \operatorname{PAR}\left(\boldsymbol{x}_{m}\right) \leq \rho, \text { for each } m, \\
\left\|\boldsymbol{x}_{m}\right\|_{2}^{2}=c, \text { for each } m .
\end{gathered}
$$

Step 3. For each $n, n=-N / 2, \ldots, N / 2-1$, update $\varphi$ based on the estimated $\boldsymbol{X}$ by solving

$$
\min _{\varphi_{n}}\left\|\boldsymbol{y}_{n} \varphi_{n}-\hat{\boldsymbol{y}}_{n}\right\|,
$$

where $\hat{\boldsymbol{y}}_{n}$ is the $n$th column of $\left[\operatorname{DFT}\left(\boldsymbol{x}_{1}\right)\right.$, $\left.\operatorname{DFT}\left(\boldsymbol{x}_{2}\right), \quad \ldots, \quad \operatorname{DFT}\left(\boldsymbol{x}_{M}\right)\right], \quad$ with $\operatorname{DFT}(\cdot)$ representing the discrete Fourier transform (DFT) operator. This minimization problem (19) has a closed-form solution:

$$
\varphi_{\mathrm{n}}=\exp \left\{j \arg \left[\boldsymbol{y}_{n}{ }^{H} \hat{\boldsymbol{y}}_{n}\right]\right\} .
$$

Step 4. Repeat steps 2 and 3 until convergence is achieved.

denoted as $D$, is larger than one. In this case, $\boldsymbol{S}\left(f_{n}\right)$ is expressed as

$$
\boldsymbol{S}\left(f_{n}\right)=\varepsilon_{n 1} \boldsymbol{y}_{n 1} \boldsymbol{y}_{n 1}^{H}+\varepsilon_{n 2} \boldsymbol{y}_{n 2} \boldsymbol{y}_{n 2}^{H}+\ldots+\varepsilon_{n D} \boldsymbol{y}_{n D} \boldsymbol{y}_{n D}^{H},
$$

where $\varepsilon_{n d}$ and $\boldsymbol{y}_{n d}$ denote the $d$ th largest eigenvalue and the corresponding eigenvector, respectively. Similar to the single-rank case as described in Section 3.1, each set of $\left\{\boldsymbol{y}_{n d}\right\}_{n=-N / 2}^{N / 2-1}$ can be used to compute a set of $\left\{\boldsymbol{Y}_{d}, \boldsymbol{Z}_{d}, \boldsymbol{X}_{d}\right\}$. Note that the eigenvalues $\varepsilon_{n d}$ is included in $\boldsymbol{y}_{n d}$ in the optimization process, for the purpose of approximately matching the optimized $\boldsymbol{S}\left(f_{n}\right)$.

It is noted that simultaneous transmission of the optimized waveforms $\boldsymbol{X}_{d}, d=1, \ldots, D$, will violate the PAR constraints. As such, the $D$ waveforms are sequentially transmitted, i.e., the transmit waveforms are expressed as

$$
\begin{aligned}
\hat{\boldsymbol{Z}} & =\left[\boldsymbol{Z}_{1}, \boldsymbol{Z}_{2}, \ldots, \boldsymbol{Z}_{D}\right]_{M \times D N}, \\
\hat{\boldsymbol{X}} & =\left[\boldsymbol{X}_{1}, \boldsymbol{X}_{2}, \ldots, \boldsymbol{X}_{D}\right]_{M \times D N} .
\end{aligned}
$$

Consequently, the low PAR waveform design problem in (15) can be modified as

$$
\begin{gathered}
\min _{\boldsymbol{X}, \boldsymbol{p}}\|\hat{\boldsymbol{Z}}-\hat{\boldsymbol{X}}\|_{F} \\
\text { s.t. } \quad \operatorname{PAR}\left(\hat{\boldsymbol{x}}_{m}\right) \leq \rho, \text { for each } m \\
\left\|\hat{\boldsymbol{x}}_{m}\right\|_{2}^{2}=c, \text { for each } m .
\end{gathered}
$$

The solution of the minimization problem in (24) follows the same iterative steps as the algorithm described in Section 3.1. The computation complexity of the proposed algorithm in general case is $O\left(M^{3}+L D M N\left(N+\log _{2} N\right)\right)$. 


\subsection{Analysis}

Unlike the WBFIT method [17], which only solves the beampattern matching problem, and the SFBT method [16], which does not design waveforms based on a given CSDM, the proposed algorithm can synthesize transmit waveforms which match any given CSDM. This enables the proposed method to design wideband MIMO radar beampatterns much more flexibly. However, the proposed method is generally suboptimal because there are distortions between the resulting beampattern and the desired one due to two factors. One is caused by the negligence of insignificant eigen-terms in eqs. (11) and (21). This error can be controlled by properly choosing the number of effective eigenvalues of $\boldsymbol{S}\left(f_{n}\right)$. A large value of $D$ yields a smaller error in (21), but will result in a long transmit sequence. The small components in $\boldsymbol{S}\left(f_{n}\right)$ will make it much more difficult to satisfy the low PAR constraint. Consequently, we should properly choose the number of primary eigenvalues, $D$, e.g., whose sum exceeds a certain percentage (say, 99\%) of the total sum of all eigenvalues. A similar strategy was utilized in [24]. On the other hand, strict low PAR constraint also results in mismatches between the optimized beampattern and the desired one, especially in multi-rank case. This can only be relieved by choosing a larger value of $\rho$ within the acceptable limit.

\section{SIMULATION RESULTS}

In this section, we provide simulation results to demonstrate the effectiveness of the proposed algorithm for wideband MIMO waveform design based on given a CSDM, which can be obtained by the optimization method described in Section 2. Throughout the simulations, a MIMO radar with a ULA of $M=16$ omni-directional antennas is assumed. The carrier frequency of the transmitted signals is $f_{c}=1 \mathrm{GHz}$, the bandwidth is set as $B=100 \mathrm{MHz}$, and $N=64$ frequencydomain samples are assumed. The inter-element spacing is set as half wavelength of the highest in-band frequency to avoid grating lobes. The spatial angle is divided into $K=181$ grid points.

In this example, the min-max sidelobe beampattern design method is adopted to design the desired beampattern pointing at $0^{\circ}$ with a main beam width of $8^{\circ}$. The sidelobe regions are set as

$$
\Theta=\left[-90^{\circ},-9^{\circ}\right] \bigcup\left[9^{\circ}, 90^{\circ}\right],-N / 2 \leq n \leq N / 2-1 .
$$

Note buffer zones with a width of $5^{\circ}$ are assumed between the $3 \mathrm{~dB}$ power points and the sidelobe regions. By solving the optimization problem in (10), we obtain the CSDM $S\left(f_{n}\right)$ at each frequency $f_{n}$. The desired beampattern computed from the optimized CSDM is shown in Fig. 1(a). In the proposed method, $D$ is chosen as 2 , and the PAR constraint is set as $\rho=2$. As shown in Fig. 1(b), the beampattern synthesized by the actual waveforms is very approximate to the desired one, only with a little distortion in the sidelobe regions. On the other hand, we also compare the proposed method with the method in [17] in the spatial-angle dimension, which is shown in Fig. 2. It is shown that our method can obtain lower sidelobe performance, because the low sidelobe constraint in CSDM optimization which cannot be solved by the method in [17].

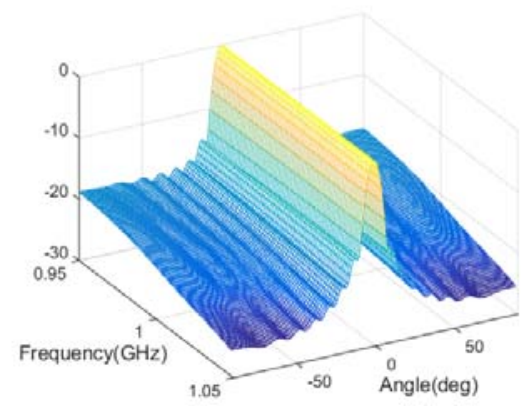

(a)

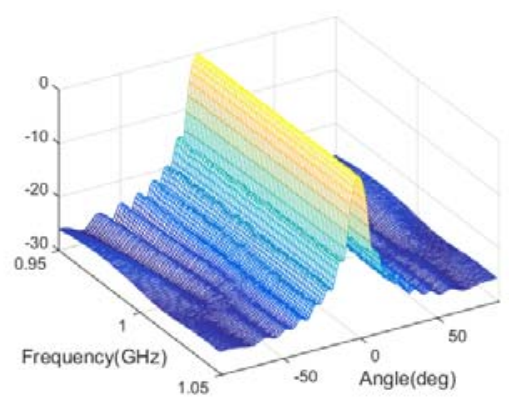

(b)

Fig 1. Beampatterns synthesized with respect to spatial angle and frequency using (a) the optimized CSDM and (b) the proposed iterative algorithm with $\rho=2$.

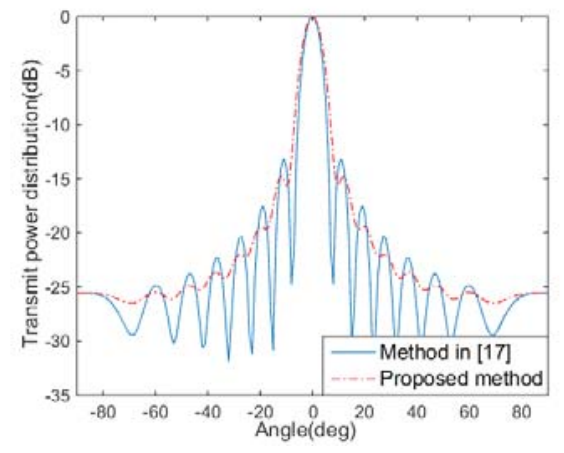

Fig. 2. Comparison of beampatterns generated by two different methods with respect to spatial angle dimension.

\section{CONCLUSION}

In this paper, we have proposed an optimization method to design wideband MIMO radar transmit waveforms based on the arbitrary CSDM. In particular, the proposed waveform design methods achieve low PAR constraints while meeting the specified CSDM. Simulation results verified the effectiveness of the proposed algorithm for designing low PAR waveforms that synthesize the desired beampatterns. 


\section{REFERENCES}

[1] E. Fishler, A. Haimovich, R. Blum, D. Chizhik, L. Cimini, and R. Valenzuela, "MIMO radar: An idea whose time has come," in Proceedings of IEEE Radar Conference, Philadelphia, PA, pp. 71-78, April 2004.

[2] J. Li and P. Stoica, "MIMO radar with co-located antenna: Review of some recent work," IEEE Signal Processing Magazine, vol. 24, pp. 106-114, 2007.

[3] M. Haimovich, R. S. Blum, and L. J. Cimini, "MIMO radar with widely separated antennas," IEEE Signal Processing Magazine, vol. 25, pp.116-129, 2008.

[4] F. Daum and J. Huang, "MIMO radar: snake oil or good idea?" IEEE Aerospace and Electronic Systems Magazine, vol. 24, pp. 8-12, 2009.

[5] J. Li, P. Stoica, and Y. Xie, "On probing signal design for MIMO radar," in Proceedings of Asilomar Conference on Signals, Systems and Computers, Pacific Grove, CA, pp. 3135, 2006.

[6] J. Li, P. Stoica, K. Forsythe, and D. Bliss, "Range compression and waveform optimization for MIMO radar: A Cramér-Rao Bound based study," IEEE Transactions on Signal Processing, vol. 56, pp. 218-232, 2008.

[7] P. Stoica, J. Li, and X. Zhu, "Waveform synthesis for diversity-based transmit beampattern design," IEEE Transactions on Signal Processing, vol. 56, pp. 2593-2598, 2008.

[8] D. R. Fuhrmann and G. S. Antonio, "Transmit beamforming for MIMO radar systems using signal cross-correlation," IEEE Transactions on Aerospace and Electronic Systems, vol. 44, pp. 171-186, 2009.

[9] P. E. Berry and D. Yau, "Optimal fast-time beamforming with linearly independent waveforms," Signal Processing, vol. 89, pp. 492-501, 2009.

[10] R. Boyer, "Co-located MIMO radar with orthogonal waveform coding : Cramér-Rao lower bound," in Proceedings of IEEE International Workshop on Computational Advances in Multi-Sensor Adaptive Processing, Aruba, Dutch Antilles, pp. 149-152, Dec. 2009.

[11] A. Hassanien and S. Vorobyov, "Phased-MIMO radar: A tradeoff between phased-array and MIMO radars," IEEE Transactions on Signal Processing, vol. 58, pp. 3137-3151, 2010.

[12] H. Li and B. Himed, "Transmit subaperturing for MIMO radars with co-located antennas," IEEE Journal of Selected Topics in Signal Processing, vol. 4, no. 1, pp. 55-65, Feb. 2010.

[13] S. Ahmed, J. S. Thompson, B. Mulgrew, and Y. Petillot,
"Unconstrained synthesis of covariance matrix for MIMO radar transmit beampattern," IEEE Transactions on Signal Processing, vol. 59, pp. 3837 -3849, 2011.

[14] S. Ahmed, J. S. Thompson, B. Mulgrew, and Y. Petillot, "Finite alphabet constant-envelope waveform design for MIMO radar," IEEE Transactions on Signal Processing, vol. 59 , pp. 5326-5337, 2011.

[15] S. G. Antonio, and D. R. Fuhrmann, "Beampattern synthesis for wideband MIMO radar systems," in Proceedings of IEEE International Workshop on Computational Advances in Multi-Sensor Adaptive Processing, pp. 105-108, 2005.

[16] P. Jardin, F. Nadal, and S. Middleton, "On wideband MIMO radar: Extended signal model and spectral beampattern design," in Proceedings of European Radar Conference, pp. 392-395, 2010.

[17] Y. Jin, X. Liu, and J. Huang, "Wideband transmit beampattern design for MIMO array," in Proceedings of IEEE International Conference on Signal Processing, Communications and Computing, pp. 1-4, 2011.

[18] H. He, P. Stoica, and J. Li, "Wideband MIMO systems: signal design for transmit beampattern synthesis," IEEE Transactions on Signal Processing, vol. 59, pp. 618-628, 2011.

[19] T. Yang, S. Tao, and Z, Wu, "Fast frequency invariant transmit beampattern synthesis for wideband MIMO radar," in Proceedings of IET International Conference on Radar Systems, Glasgow, UK, pp. 52-52, Oct. 2012.

[20] W. Liu and W. Stephan, "Design of frequency invariant beamformers for broadband arrays," IEEE Transactions on Signal Processing, vol.56, pp. 855-860, 2008.

[21] J. A. Tropp, I. S. Dhillon, R. W. Heath, and T. Stroh, "CDMA signature sequences with low peak-to-averagepower ratio via alternating projection," in Proceedings of Asilomar Conference on Signals, Systems and Computers, Monterrey, CA, Nov. 2003.

[22] H. He, P. Stoica, and J. Li, "Designing unimodular sequence sets with good correlations-Including an application to MIMO radar," IEEE Transactions on Signal Processing, vol. 57, no. 11, pp. 4391-4405, Nov. 2009.

[23] J. A. Tropp, I. S. Dhillon, R. W. Heath, and T. Strohmer, "Designing structured tight frames via an alternating projection method," IEEE Transactions on Information Theory, vol. 51, pp.188-209, 2005.

[24] A. Hassanien and S. Vorobyov, "Transmit energy focusing for DOA estimation in MIMO radar with collocated antennas," IEEE Transactions on Signal Processing, vol. 59, pp.2669-2682, 2011. 